\title{
ARTICLE OPEN \\ Reaction-diffusion theory explains hypoxia and heterogeneous growth within microbial biofilms associated with chronic infections
}

\author{
Philip S Stewart ${ }^{1,2}$, Tianyu Zhang ${ }^{1,3}$, Ruifang $\mathrm{Xu}^{1,2}$, Betsey Pitts ${ }^{1}$, Marshall C Walters ${ }^{1,2,5}$, Frank Roe ${ }^{1,6}$, Judith Kikhney ${ }^{4}$ \\ and Annette Moter ${ }^{4}$
}

Reaction-diffusion models were applied to gain insight into the aspects of biofilm infection and persistence by comparing mathematical simulations with the experimental data from varied bacterial biofilms. These comparisons, including three in vitro systems and two clinical investigations of specimens examined ex vivo, underscored the central importance of concentration gradients of metabolic substrates and the resulting physiological heterogeneity of the microorganisms. Relatively simple one-dimensional and two-dimensional (2D) models captured the: (1) experimentally determined distribution of specific growth rates measured in Pseudomonas aeruginosa cells within sputum from cystic fibrosis patients; (2) pattern of relative growth rate within aggregates of streptococcal biofilm harboured in an endocarditis vegetation; (3) incomplete penetration of oxygen into a Pseudomonas aeruginosa biofilm under conditions of exposure to ambient air and also pure oxygen; (4) localisation of anabolic activity around the periphery of $P$. aeruginosa cell clusters formed in a flow cell and attribution of this pattern to iron limitation; (5) very low specific growth rates, as small as $0.025 \mathrm{~h}^{-1}$, in the interior of cell clusters within a Klebsiella pneumoniae biofilm in a complex 2D domain of variable cell density.

npj Biofilms and Microbiomes (2016) 2, 16012; doi:10.1038/npjbiofilms.2016.12; published online 22 June 2016

\section{INTRODUCTION}

The list of chronic infections stemming from biofilms continues to grow as does awareness of the economic and human toll incurred by these debilitating infections. Reaction-diffusion theory has been successfully applied for decades to understand microscale chemical gradients, ecological niches and substrate fluxes in wastewater treatment and environmental biofilms. There are far fewer examples of the adaptation of this theory to systems of medical relevance. Here the general utility of this class of models, which derive from first principles of concerted Fickian diffusion and metabolic reaction, is illustrated with case studies involving limitation for oxygen, glucose and iron. Recurrent themes of hypoxia, physiological heterogeneity in the microbial population and antibiotic tolerance resulting from non-growing cells emerge. Altogether, these examples show that reaction-diffusion theory can be applied to shed light on the chemical and physiological heterogeneity that likely contributes to the pathogenesis and persistence of biofilm infections.

Mathematical modelling of biofilms has contributed to the understanding of the functioning of these aggregated microbial communities since the seminal article of Enrique Lamotta in 1976. ${ }^{1}$ Hundreds of biofilm modelling papers have been published subsequently, of which we cite here only a few of the landmark examples. ${ }^{2-9}$ Almost all of these studies were motivated by applications in water and wastewater treatment. They address the operation of systems such as trickling filtres, anaerobic sludge digesters and drinking water distribution pipes.

As the awareness of the role of biofilm formation in numerous persistent infections expands, ${ }^{10-13}$ an opportunity exists to enhance the understanding of the formation, activity and ecology of infectious biofilms by adapting the same types of mathematical models that have been so successful in civil engineering application to the systems of medical and dental interest. This opportunity remains relatively unexplored and underdeveloped. There are a few pioneering examples of biofilm modelling applied to medical systems. ${ }^{14-24}$ These models have contributed important insights into such topics as the mechanism of dental caries, ${ }^{15,23}$ the penetration of antibiotics into biofilms, ${ }^{14,17}$ the induction of quorum sensing in a biofilm ${ }^{19}$ and probiotic control of a pathogenic biofilm. ${ }^{22}$

The purpose of the work reported in this article is to demonstrate the general applicability of reaction-diffusion theory to biofilm problems of medical relevance. We focus in particular on the capacity of this theoretical approach to predict gradients in the concentration of metabolic substrates and, as a consequence, patterns of microbial growth that are spatially heterogeneous.

${ }^{1}$ Center for Biofilm Engineering, Montana State University, Bozeman, MT, USA; ${ }^{2}$ Chemical and Biological Engineering, Montana State University, Bozeman, MT, USA; ${ }^{3}$ Mathematical Sciences, Montana State University, Bozeman, MT, USA and ${ }^{4}$ Biofilmcenter, German Heart Institute Berlin, Berlin, Germany.

Correspondence: PS Stewart (phil_s@biofilm.montana.edu)

${ }^{5}$ Current address: Cargill Malt, Spiritwood, ND, USA

${ }^{6}$ Current address: Davis, CA, USA

Received 7 December 2015; revised 11 April 2016; accepted 19 May 2016 


\section{RESULTS}

Case 1-prediction of the spatial distribution of oxygen within a biofilm and how it depends on the concentration of oxygen applied to the surface of the biofilm

Because oxygen is sparingly soluble and is rapidly respired by aerobic microorganisms, oxygen concentration gradients are a common feature of biofilm systems. The oxygen profile measured in a simple in vitro $P$. aeruginosa biofilm describes a curve whose shape is fit reasonably well by the parabolic solution to the zeroorder reaction-diffusion problem (Figure 1a). In this example, a value of the Thiele modulus of $\phi_{\mathrm{o}}=2.82$ provides an acceptable description of the data (Figure $1 \mathrm{~b}$ ). The experimental measurements plotted in Figure 1 are from a single-colony biofilm that was first profiled in air then flooded with pure oxygen gas and profiled again in this oxygen-enriched environment. The measurements were performed in the same exact planar location in the biofilm. Thus, the fixed physical $\left(L_{\mathrm{f}}, \rho, D_{\mathrm{e}}\right)$ and intrinsic biological parameters $\left(\mu_{\mathrm{o}}, Y_{\mathrm{xs}}\right)$ that constitute $\phi_{\mathrm{o}}$ were identical. The only difference between the air and oxygen conditions was owing to the change in the concentration of oxygen at the boundary $\left(C_{0}\right)$. We predict therefore that the ratio of $\phi_{\mathrm{o}}$ values between these two cases should be numerically equal to the square root of the ratio of the bulk oxygen concentrations (see Equation (4)), which is $(28.5 / 6)^{1 / 2}$ or 2.18 . The actual ratio of the two fitted values of
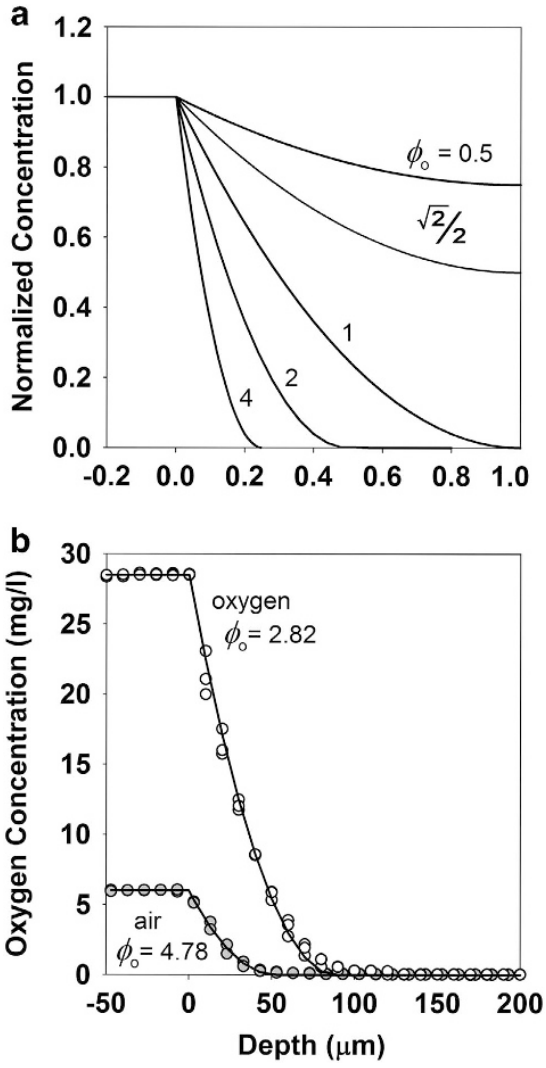

Figure 1. Experimental and theoretical oxygen concentration profiles in a $P$. aeruginosa colony biofilm. (a) Theoretical concentration profiles (solid curves) for a metabolic substrate experiencing zero-order reaction kinetics for varying values of the Thiele modulus, $\phi_{\mathrm{o}}$. (b) Experimental oxygen concentration profiles in a single $P$. aeruginosa colony biofilm exposed to either air (grey circles) or oxygen-enriched gas (open circles). The solid lines are theoretical curves fit to the two data sets. $\phi_{\text {o }}$ was $4.78 / 2.82$ or 1.70 . The Thiele modulus values determined in air and oxygen were statistically significantly different $\left(P<10^{-4}\right)$.

Case 2-calculation of the probability distribution of specific growth rates in a biofilm

Given that biofilms harbour gradients in the concentration of the growth-limiting substrate, there will also be corresponding changes in the local cellular specific growth rate. For the case of first-order reaction kinetics this leads to variation in the growth rate in space that is captured in the distributions plotted in Figure $2 a$. When diffusion is only slightly limiting $\left(\phi_{1}=0.5\right)$ growth rates are rapid and close to the growth rate that would be expected with the bulk fluid concentration of substrate $\left(\sim 1 \mathrm{~h}^{-1}\right)$. As diffusion limitation increases ( $\phi_{1}$ progressively larger) the distribution becomes broader and includes more lower values of growth rate.

Kragh et al. ${ }^{25}$ recently reported single-cell measurements of specific growth rate of $P$. aeruginosa in the sputum of three patients with cystic fibrosis. We pooled these 63 measurements and created a quadripartite distribution. The theoretical distribution for a value of $\phi_{1}=5$ matches this experimental result well (Figure 2b).
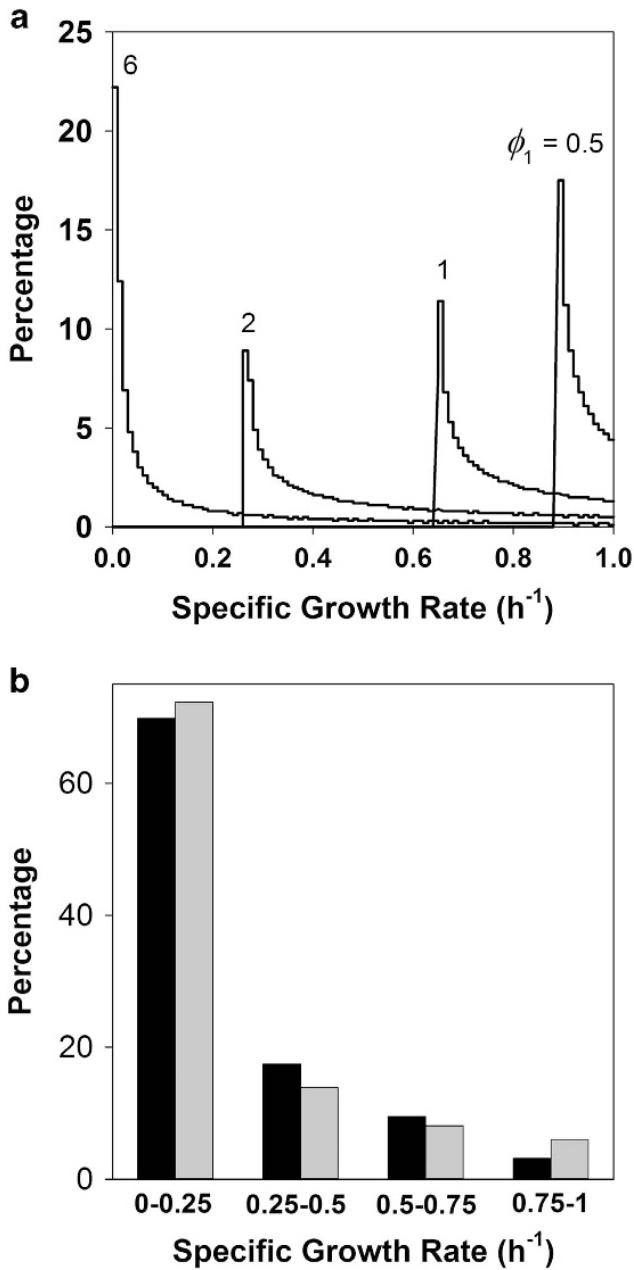

Figure 2. Predicted distribution of bacterial growth rates in a biofilm. (a) Growth rate distributions for a flat slab biofilm governed by first-order reaction kinetics for varying values of the Thiele modulus, $\phi_{1}$. (b) Comparison of measured growth rate distribution of $P$. aeruginosa in the explanted lungs of three CF patients ${ }^{25}$ (black bars) with the distribution predicted for $\phi_{1}=5$ (grey bars). 

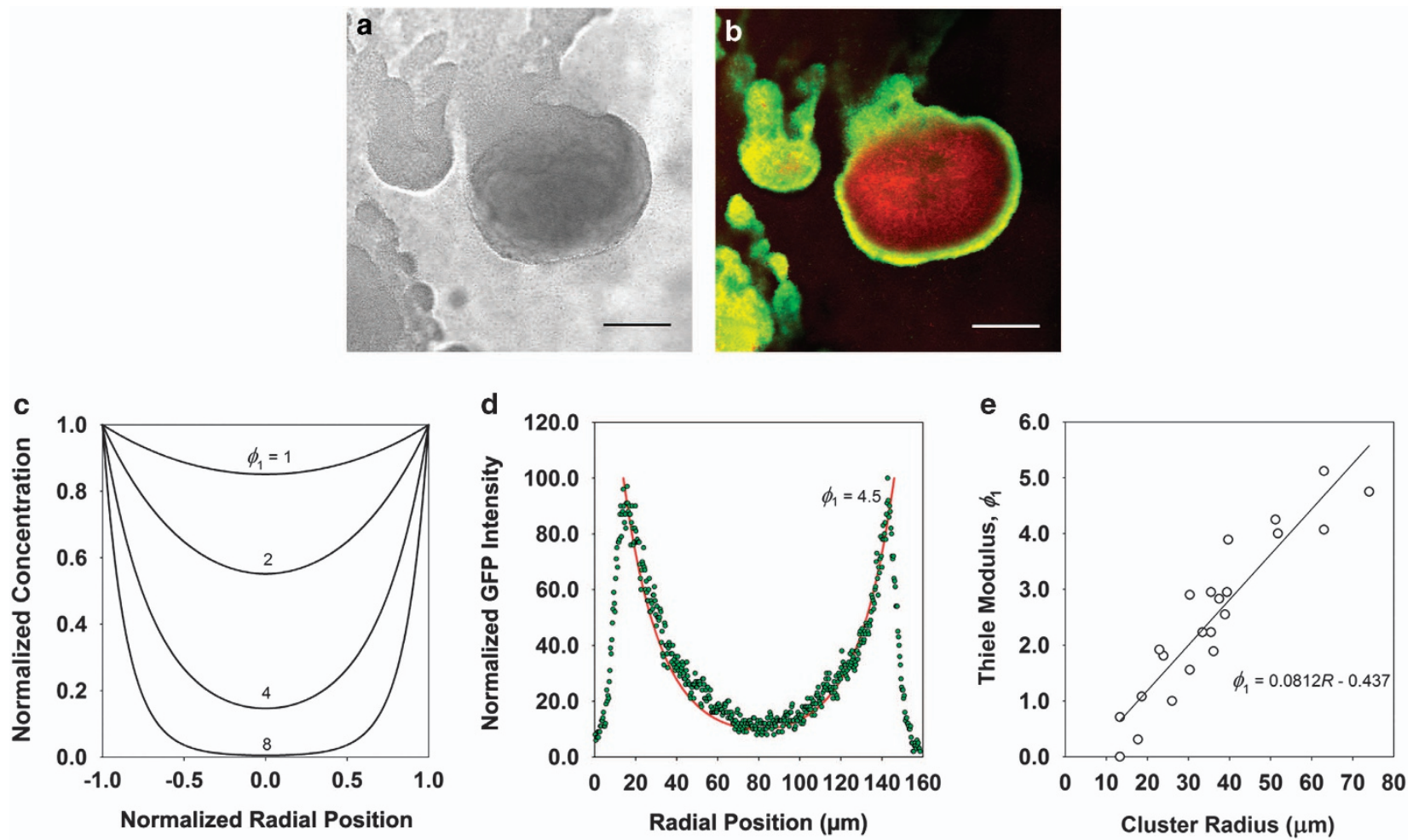

Figure 3. Relative growth rate patterns in round clusters of $P$. aeruginosa biofilm. (a) Transmission image of a cell cluster. (b) Pattern of GFP induction (green) and biomass counterstain (red) in the same cell cluster. $\mathbf{a}$ and $\mathbf{b}$ were imaged through the glass attachment surface in the plane of the substratum scale bars are 50 microns. (c), Theoretical substrate concentration profiles in a hemispherical cluster subject to firstorder reaction kinetics for varying values of the Thiele modulus, $\phi_{1}$. (d) Theoretical curve $\left(\phi_{1}=4.5\right)$ fitted to an experimental GFP fluorescence intensity obtained by image analysis from an experiment like that shown in b. (e) Thiele modulus determined by fit to experimental data plotted versus cluster radius (symbols). The line is a least squares regression whose slope has the value $\left(k_{1} / D_{\mathrm{e}}\right)^{1 / 2}$; see Equation $(17)$.

Case 3-visualisation and quantification of a growth rate gradient within a biofilm and identification of the growth-limiting substrate as iron

One way to visualise gradients in anabolic activity within a biofilm is by using a bacterial strain containing an inducible fluorescent protein. A time-lapse microscopy video sequence of such an experiment can be viewed in the Supplementary Movie S1. In this experiment, a $P$. aeruginosa biofilm containing an isopropylthio$\beta$-D-galactoside-inducible green fluorescent protein (GFP) was grown for 5 days in the absence of the inducer. The biofilm was dark at this point. The inducing agent was then added to the medium, in uninterrupted continuous flow. Over the next few hours, green colour developed in the cell cluster corresponding to the local expression of GFP. More GFP was expressed at the periphery of the cluster than at the cluster center. This gradient reflects the relative growth rate of the bacteria in different regions of the cluster.

The process of quantifying the pattern of growth within an experiment like that described above is illustrated in Figure 3. A biofilm cluster imaged in transmission mode was $\sim 126 \mu \mathrm{m}$ in diameter (Figure 3a). After the induction of GFP and counterstaining with a red dye, a pattern of GFP expression similar to that described above was observed: brighter green near the edges of the cluster and dimmer green towards the center (Figure 3b). The red stain reveals the distribution of biomass independent of metabolic activity. Calculated concentration profiles (Equation (18)) of a reacting substrate in a hemispherical cluster are given in Figure $3 c$ for several values of the Thiele modulus, $\phi_{1}$. Note that as the growth rate in this case is directly proportional to the local substrate concentration, the spatial patterns calculated in
Figure $3 c$ are expected to apply to the microbial-specific growth rate as well as the substrate concentration itself. Image analysis of the green fluorescence intensity within a biofilm cluster can be compared with the theoretical patterns like those in Figure $3 \mathrm{c}$ to extract a quantitative estimate of the Thiele modulus. An example of such a fit is shown in Figure 3d.

When this image analysis process was applied to multiple biofilm clusters $(n=22)$ from several experiments, a range of values of $\phi_{1}$ were determined (Figure 3e). The estimated Thiele modulus increased with the measured radius of the cell cluster (Figure 3e). This linear dependence is expected from the definition of the Thiele modulus. The slope of the line fitted to the data in Figure $3 e$ provides access to a quantitative estimate of the value of the first-order reaction rate coefficient, $k_{1}$.

This discussion has so far not addressed the identity of the growth-limiting substrate. Indeed, the observation of an activity gradient within a biofilm does not by itself provide any clue as to the nature of the limitation. The likely identity of the growthlimiting substrate can be accessed by comparing values of $k_{1}$ derived from experiment (i.e., Figure $3 \mathrm{e}$ ) and calculated a priori from independent estimates of the constituent parameters. These comparisons are summarised in Table 1. All of the theoretical estimates made use of the same value of the growth rate of the bacteria under bulk fluid conditions $\left(\mu_{\mathrm{o}}\right)$. The growth rate at $23^{\circ} \mathrm{C}$ of this $P$. aeruginosa strain in the minimal medium used was measured in batch culture to be $0.16 \mathrm{~h}^{-1}$. The theoretical estimates also used the same constant value of the cell density in the biofilm $(\rho)$ of $10^{4} \mathrm{mg} \mathrm{I}^{-1}$. Yield coefficients $\left(Y_{\mathrm{xs}}\right)$ were estimated based on the typical composition of biomass and reference to measured values. ${ }^{26}$ The bulk fluid concentration of the limiting substrate $\left(C_{0}\right)$ was determined by the medium 
composition for carbon and nitrogen and by the solubility of oxygen in water at the typical barometric pressure in Bozeman, Montana. Iron concentration was estimated as the solubility limit of iron(III) phosphate. ${ }^{27}$ Diffusion coefficients in the biofilm were estimated as described elsewhere. ${ }^{28,29}$ Comparison of the experimental values of $k_{1}$ with the calculated theoretical values reveals a discrepancy for carbon, nitrogen and oxygen of two orders of magnitude or more. Only for iron is there a reasonable correspondence. Iron is thus the likely growth-limiting substrate in this case.

Case $4 \mathrm{~A}$-calculation of spatial variation in growth rate within a heterogeneous biofilm in vitro

Wentland et al. $^{30}$ reported an experimental visualisation of the relative growth rate within a $K$. pneumoniae biofilm grown in vitro in a continuous flow reactor. This result is reproduced in Figure $4 a$.

Table 1. Comparison of experimental $\left(k_{1, \text { ex }}\right)$ and theoretical $\left(k_{1, \text { th }}\right)$ values of the first-order reaction rate coefficient for different limiting substrates in a $P$. aeruginosa biofilm

\begin{tabular}{lccccc}
\hline $\begin{array}{l}\text { Limiting } \\
\text { substrate }\end{array}$ & $\begin{array}{c}D_{e} \\
\left(\mathrm{~cm}^{2} \mathrm{~s}^{-1}\right)\end{array}$ & $\begin{array}{c}k_{1, \text { ex }} \\
\left(\mathrm{s}^{-1}\right)\end{array}$ & $\left(g_{x} / g_{s}\right) Y_{x s}$ & $\begin{array}{c}C_{o} \\
\left(\mathrm{mg} \mathrm{l}^{-1}\right)\end{array}$ & $\begin{array}{c}k_{1, \text { th }} \\
\left(\mathrm{s}^{-1}\right)\end{array}$ \\
\hline $\mathrm{C}$ & $2.23 \times 10^{-6}$ & 1.47 & 0.25 & 180 & 0.010 \\
$\mathrm{~N}$ & $1.80 \times 10^{-6}$ & 1.19 & 7.1 & 12.6 & 0.005 \\
$\mathrm{O}_{2}$ & $11.4 \times 10^{-6}$ & 7.52 & 0.85 & 6.0 & 0.087 \\
$\mathrm{Fe}$ & $3.41 \times 10^{-6}$ & 2.25 & 500 & 0.00043 & 2.07 \\
\hline
\end{tabular}

Substrates are glycerol $(\mathrm{C})$, glutamate $(\mathrm{N})$, molecular oxygen $\left(\mathrm{O}_{2}\right)$ and $\mathrm{Fe}^{2+}$ $(\mathrm{Fe})$. The medium concentrations, $C_{0}$, are as the element. Diffusion coefficients in the biofilm were estimated as described elsewhere. ${ }^{52,53}$ Yield coefficients $\left(Y_{\mathrm{xs}}\right)$ were estimated based on the typical composition of biomass and reference to measured values. ${ }^{50}$
The biofilm varied in thickness and local cell density. There was a band of active growth (indicated by orange or red colour) that tracked the biofilm-bulk fluid interface. Where the biofilm was thinner and less dense, the entire biofilm thickness was growing rapidly as indicated by the hotter colours. Where the biofilm was locally thicker, the interior of the clusters suggested lower growth rates as indicated by the cooler colours of yellow and green.

We simulated the distribution of the growth-limiting substrate, glucose, within a two-dimensional representation of the biofilm using parameter values summarised in Supplementary Table S2. An example of the predicted glucose concentration is shown in Figure $4 \mathrm{~b}$. This simulation shows that glucose concentrations are diminished within the three larger biofilm clusters. As the bulk fluid glucose concentration was varied (this being the one parameter for which an independent experimental estimate was not available), the predicted pattern of growth rates within the biofilm changed. At a relatively low bulk concentration of $10 \mathrm{mgl}^{-1}$, growth rates in the biofilm were also relatively slow as indicated by a predominance of green and yellow hues (Figure 4c). At the highest bulk glucose concentration simulated, $40 \mathrm{mg} \mathrm{I}^{-1}$, growth rates were higher and much of the biofilm was predicted to be growing rapidly (Figure 4e). As the reactor influent concentration of glucose was $40 \mathrm{mg} \mathrm{I}^{-1}$, this constitutes an upper bound to the actual concentration experienced by the biofilm. The simulation at a bulk fluid glucose concentration of $25 \mathrm{mg} \mathrm{I}^{-1}$ (Figure 4d) resembles the experimental pattern (Figure 4a). Predicted growth rates manifested as a clear spatial gradient within the biofilm ranging from $0.71 \mathrm{~h}^{-1}$ (84\% of the maximum growth rate) to just $0.025 \mathrm{~h}^{-1}$ (3\% of maximum).

Case $4 \mathrm{~B}$ - calculation of spatial variation in growth rate within a heterogeneous biofilm ex vivo

We analyse here the pattern of activity found in a fluorescence in situ hybridisation (FISH)-probed infectious biofilm recovered
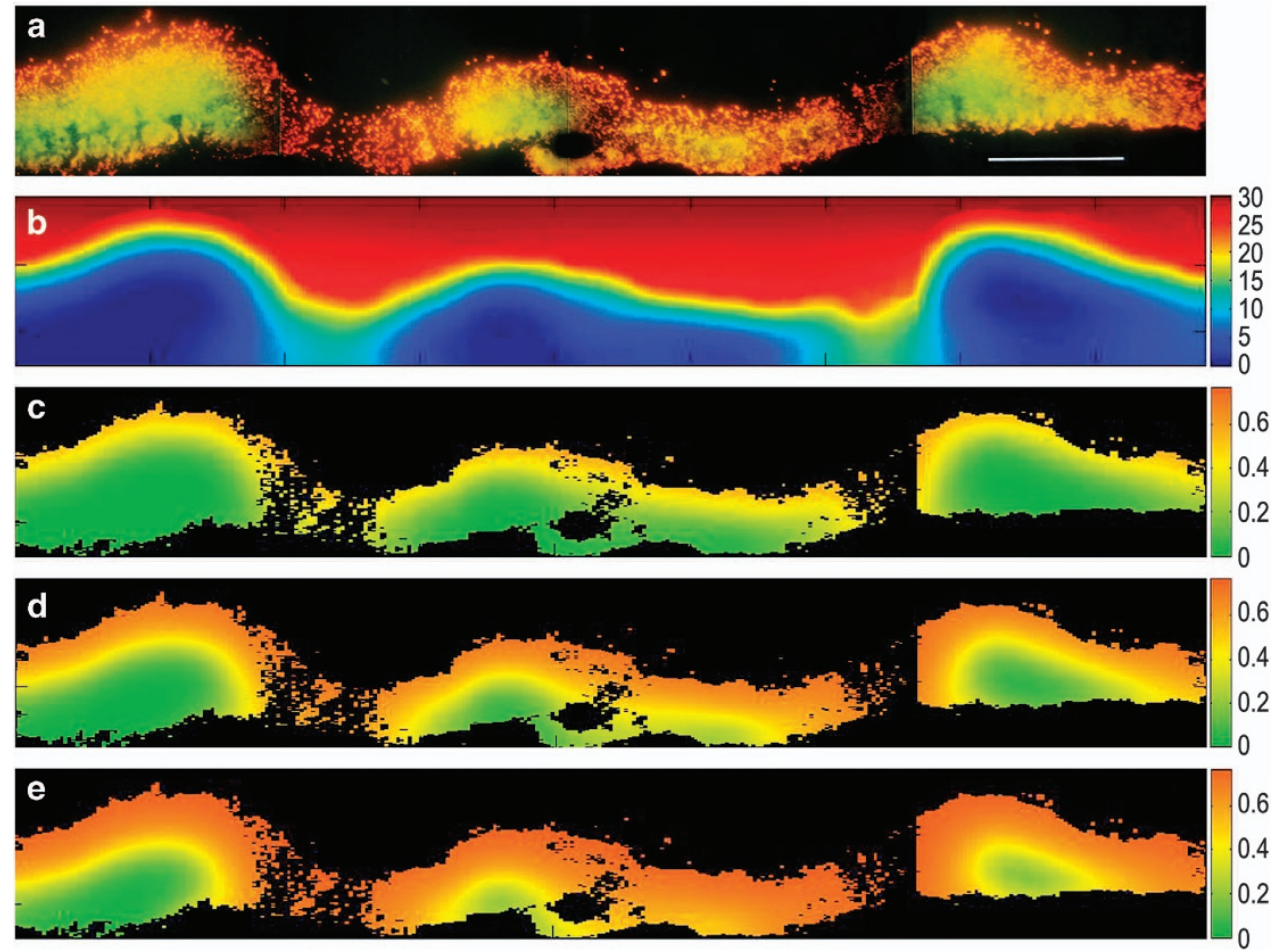

Figure 4. Simulated spatial patterns of specific growth rate in a 2D heterogeneous biofilm structure compared with an in vitro result. (a) Acridine orange stained frozen section showing regions of relatively rapid (red/orange) and slow (green/yellow) growth (reprinted with permission from ref. 30). (b) Calculated glucose concentration $\left(\mathrm{mg} \mathrm{I}^{-1}\right.$ ) for a bulk fluid glucose concentration of $30 \mathrm{mg} \mathrm{I}^{-1}$. (c-e) The predicted specific growth rate $\left(\mathrm{h}^{-1}\right)$ for bulk fluid glucose concentrations of $10 \mathrm{mg} \mathrm{I}^{-1}$, (c); $25 \mathrm{mg} \mathrm{I}^{-1}$, (d); and $40 \mathrm{mg} \mathrm{I}{ }^{-1},(\mathbf{e})$. Bar $=100 \mu \mathrm{m}$. 

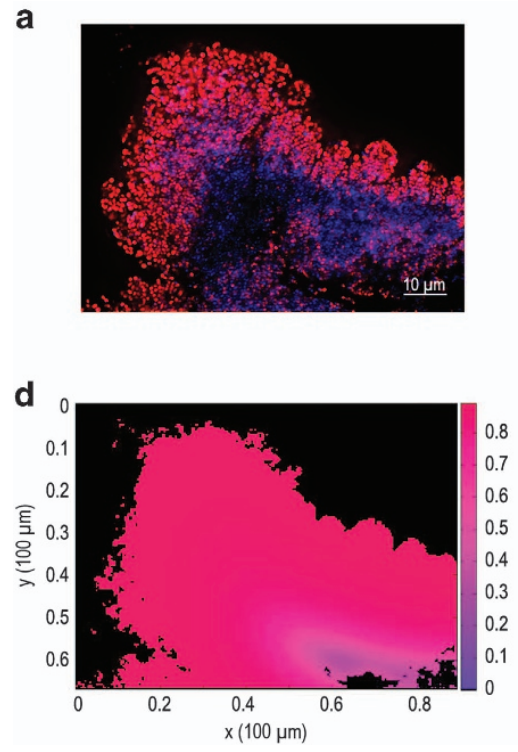

b
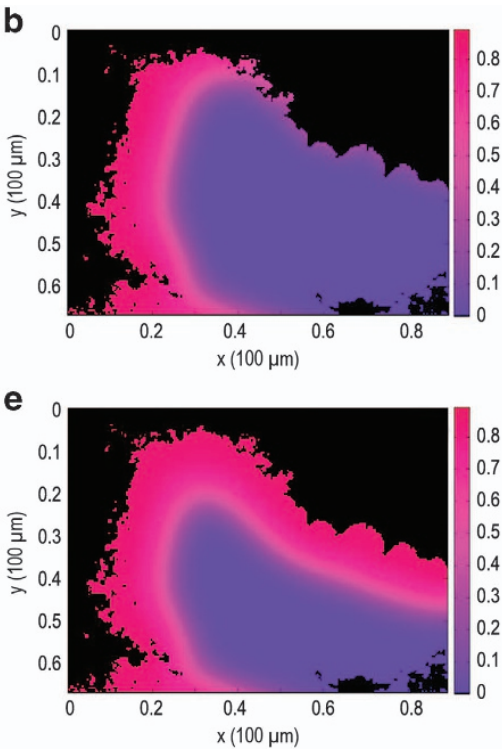
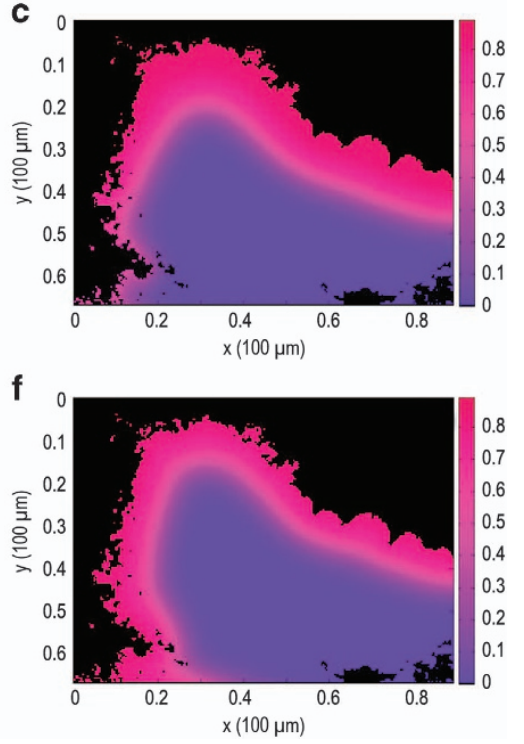

Figure 5. Simulated spatial patterns of specific growth rate in a 2D heterogeneous biofilm structure compared with an ex vivo result. (a) FISHprobed human clinical endocarditis specimen showing cells with relatively rapid (red), intermediate (magenta), or slow or no activity (blue) as indicated by relative ribosome content. (b-f) The predicted specific growth rate $\left(\mathrm{h}^{-1}\right)$ for bulk fluid glucose concentrations of 100 mg $\mathrm{I}^{-1}$, $(\mathbf{b}, \mathbf{c}, \mathbf{e}) ; 500 \mathrm{mg} \mathrm{I}^{-1},(\mathbf{d})$; and $50 \mathrm{mg} \mathrm{I}^{-1}$, (f). Glucose was provided at the left boundary (b), top boundary (c), or on both the left and top boundaries $(\mathbf{d}-\mathbf{f})$.

from the heart valve vegetation of an endocarditis patient (Figure 5a). The microorganism was determined to be Streptococcus equinus using $16 \mathrm{~S}$ rRNA (ribosomal RNA)-gene sequencing. The relative ribosome content as revealed by the fluorescence signal intensity associated with the amount of hybridised FISH probe (red colour) indicated localised activity associated with the left and top boundaries of the biofilm. Spatial patterns of bacterial growth within this structure were simulated using parameter values summarised in Supplementary Table S3. Simulations that only modelled provision of glucose from the left or top boundaries alone (Figures $5 b, c$, respectively) were unable to produce qualitatively accurate representations of the observed activity pattern. Rather, it was necessary to model the provision of the growth-limiting nutrient, glucose, from both the left and top boundaries (Figures $5 d-f$ ).

A starting point for setting the glucose concentration at the boundaries was the glucose concentration in human plasma, $\sim 900 \mathrm{mgl}^{-1}$ (ref. 31). When high concentrations of glucose were assumed along the left and top boundaries of the aggregate, rapid microbial growth was predicted throughout the biofilm (Figure 5d). Only when the boundary-condition glucose concentration was reduced to $\sim 100 \mathrm{mg} \mathrm{I}^{-1}$ or less (Figures $5 \mathrm{e}$ and f), did the predicted patterns conform to the observed pattern. This result shows that this biofilm aggregate was likely exposed to lower concentrations of nutrient than are available in plasma, probably as a result of external mass transfer limitations.

\section{DISCUSSION}

Reaction-diffusion theory can be applied to shed light on the chemical and physiological heterogeneity that likely contributes to the pathogenesis and persistence of biofilm infections. Here we have analysed elementary problems that illustrate the propensity for hypoxia in the vicinity of a biofilm, the potential for limitation by diverse substrates from oxygen to glucose to iron, and the reality of distributed growth states in the microbial population that range from rapidly growing to dormant.

The comparison reported in Figure $1 \mathrm{~b}$ can be regarded as a test of the potential of hyperbaric oxygen therapy to improve the penetration of oxygen into a biofilm. The penetration depth of oxygen is predicted to increase as the square root of the applied oxygen concentration. For example, quadrupling the oxygen tension only doubles the penetration depth of oxygen. This result highlights a possible limitation to the efficacy of delivering oxygen into an infectious biofilm with hyperbaric oxygen therapy. ${ }^{32}$ This limitation may not be widely recognised in the medical field even though the concentration dependence of substrate penetration has been known for decades in the context of biofilm wastewater treatment process. ${ }^{33}$

Hypoxia is a recurrent theme in biofilm infections. ${ }^{34-36}$ It impacts healing, the oxidative burst of neutrophils and bacterial antibiotic tolerance. ${ }^{37}$ Reaction-diffusion models are an appropriate approach in analysing hypoxia, though they will need to go beyond the simple models presented here to incorporate oxygen transport in the vasculature and oxygen consumption by host tissue and leukocytes.

Quantitative results derived from a reaction-diffusion analysis allowed us to diagnose an example of iron limitation (Figure 3). Iron has not previously been identified as a limiting substrate for biofilm growth to our knowledge. Iron limitation is quite plausible in vivo, however, where most iron is sequestered. ${ }^{38}$ The very simple minimal medium that was used in this experiment, unlike many laboratory media, contained no added trace elements. The only iron present probably entered as a contaminant of the constituent salts. Because of the relatively high concentration of phosphate, iron would have precipitated as an iron phosphate. Precipitated iron that remains in suspension will be accessible to planktonic cells, but in the biofilm only the dissolved iron would be able to access the interior of a cell cluster by diffusion.

Our calculations show that microscale biological heterogeneity in specific growth rate can be predicted from first principles of reaction and diffusion (Figures 2, 4 and 5). This heterogeneity is evident in the variation in growth rate between individual cells in a population (Figure 2 ) and in spatial patterns across multicellular aggregates of bacteria (Figures 4 and 5). In these three examples - cystic fibrosis sputum, an in vitro Enterobacterial biofilm and a bacterially colonised endocarditis vegetation-the biofilm not only harbours growing bacterial cells, but also cells that are 
growing slowly or not at all. This result may aid in understanding antibiotic tolerance arising from biofilms that harbour a significant proportion of non-growing bacteria. ${ }^{39}$

\section{MATERIALS AND METHODS}

\section{Theory}

The solutions for Cases 1, 2, and 3 are well-known in the technical literature. ${ }^{40,41}$ We recap them here and pose them in terms of microbiological and biofilm parameters. Nomenclature and units are provided in Supplementary Table S1.

\section{Case 1}

Consider a flat slab biofilm of uniform thickness in which the growthlimiting substrate is consumed subject to zero-order kinetics. The spatial variable, $z$, is oriented such that $z=0$ at the biofilm-bulk fluid interface and $z=L_{f}$ corresponds to the substratum or attachment surface. The dimensionless spatial variable is $\xi=z / L_{f}$. A differential balance on this substrate, when non-dimensionalised, yields:

$$
\frac{\mathrm{d}^{2} u}{\mathrm{~d} \xi^{2}}=2 \phi_{\mathrm{o}}^{2}
$$

where the two terms correspond to reaction (right) and diffusion (left) and $u$ denotes dimensionless substrate concentration. Boundary conditions impose a fixed concentration of substrate at the surface of the biofilm and a no-flux condition at the point in the biofilm at which substrate is depleted:

$$
\begin{aligned}
& u=1 \text { at } \xi=0 \\
& \frac{\mathrm{d} u}{\mathrm{~d} \xi}=0 \text { at } \xi=\frac{a}{L_{\mathrm{f}}}
\end{aligned}
$$

The dimensionless parameter $\phi$, termed the Thiele modulus, is a measure of the relative rates of reaction and diffusion. Because the particular form of $\phi$ depends on the kinetic form of the reaction, we use a subscript to denote the kinetic form to avoid confusion. For the zero-order kinetic problem, the Thiele modulus, interpreted in terms of microbial growth and substrate consumption in a biofilm, is

$$
\phi_{\mathrm{o}}=\left(\frac{\mu_{\max } \rho L_{f}^{2}}{2 C_{\mathrm{o}} Y_{\mathrm{xs}} D_{\mathrm{e}}}\right)^{1 / 2}
$$

The solution (the concentration profile within the biofilm) is given by

$$
u=\left\{\begin{array}{c}
\phi_{0}^{2} \xi^{2}-2 \phi_{0} \xi+1, \quad 0 \leq \xi \leq \frac{1}{\phi_{\circ}} \\
0, \quad \frac{1}{\phi_{0}} \leq \xi \leq 1
\end{array}\right.
$$

and the penetration depth of the substrate is

$$
\begin{aligned}
& a=\frac{L_{f}}{\phi_{\mathrm{o}}}, \\
& a \propto C_{\mathrm{o}}^{\frac{1}{2}} .
\end{aligned}
$$

Note that this solution applies when the biofilm is thick enough that the substrate is completely depleted at some point $\left(\phi_{\mathrm{o}}>1\right)$. A different solution applies for biofilms that are fully penetrated by the substrate:

$$
u=\phi_{0}^{2} \xi^{2}-2 \phi_{\circ}^{2} \xi+1 \text {. }
$$

\section{Case 2}

Consider a flat slab biofilm of uniform thickness in which the growthlimiting substrate is consumed subject to first-order kinetics. The microbial growth rate is proportional to the substrate concentration: $\mu=\mu_{\circ} u$. Note in this case that the spatial variable, $z$, runs in the opposite direction from the analysis in Case 1. Here $z=0$ corresponds to the bottom of the biofilm or substratum and $z=L_{\mathrm{f}}$ corresponds to the biofilm-bulk fluid interface. The non-dimensionalised spatial variable is $\xi=z / L_{\mathrm{f}}$. The differential balance on substrate, in non-dimensional form, is

$$
\frac{\mathrm{d}^{2} u}{\mathrm{~d} \xi^{2}}=\phi_{1}^{2} u
$$

with boundary conditions

$$
\begin{aligned}
& u=1 \text { at } \xi=1 \\
& \frac{\mathrm{d} u}{\mathrm{~d} \xi}=0 \text { at } \xi=0 .
\end{aligned}
$$

These boundary conditions impose a fixed concentration at the surface of the biofilm and a no-flux condition at the substratum.

The Thiele modulus is given by

$$
\phi_{1}=\left(\frac{\mu_{\mathrm{o}} \rho L_{f}^{2}}{C_{\mathrm{o}} Y_{\mathrm{xs}} D_{\mathrm{e}}}\right)^{1 / 2} \text {. }
$$

And the concentration profile is given by

$$
u=\frac{\cosh \left(\phi_{1} \xi\right)}{\cosh \phi_{1}} \text {. }
$$

Growth rates calculated from this concentration profile were binned to construct growth rate distributions.

\section{Case 3}

Consider a hemispherical biofilm cluster in which the growth-limiting substrate is consumed subject to first-order kinetics. The spatial variable, $r$, runs from $r=0$ at the center of the cluster to $r=R$ at the surface of the cluster of biofilm-bulk fluid interface and is made dimensionless with $\xi=r / R$. The differential balance on substrate, in non-dimensional form is

$$
\frac{1 \mathrm{~d}}{\xi^{2} \mathrm{~d} \xi}\left(\xi^{2} \frac{\mathrm{d} u}{\mathrm{~d} \xi}\right)=\phi_{1}^{2} u
$$

with boundary conditions,

$$
\begin{aligned}
& u=1 \text { at } \xi=1 \\
& \frac{\mathrm{d} u}{\mathrm{~d} \xi}=0 \text { at } \xi=0
\end{aligned}
$$

which impose a fixed concentration at the surface of the biofilm and a finite concentration at the cluster center, respectively.

The Thiele modulus in this case is given by

$$
\phi_{1}=\left(\frac{k_{1} R^{2}}{D_{\mathrm{e}}}\right)^{1 / 2}=\left(\frac{\mu_{\mathrm{o}} \rho R^{2}}{C_{\mathrm{o}} Y_{\mathrm{xs}} D_{\mathrm{e}}}\right)^{1 / 2} \text {. }
$$

And the solution is

$$
u=\frac{R \sinh \left(\phi_{1} \xi\right)}{r \sinh \left(\phi_{1}\right)} .
$$

\section{Case 4}

In the preceding three cases, the cell density and effective diffusion coefficient have been assumed to be constant and uniform in space and simple (one-dimensional) geometries have been assumed. Now we relax these constraints to analyse heterogeneous biofilm structures like those that occur in actual biofilms formed in nature, in vitro, or in vivo. ${ }^{42,43}$ In a two-dimensional field of width $X$ and height $Y$, the unsteady differential balance on substrate gives

$$
\frac{\partial C}{\partial t}=\frac{\partial}{\partial x}\left(D \frac{\partial C}{\partial x}\right)+\frac{\partial}{\partial y}\left(D \frac{\partial C}{\partial y}\right)-\frac{\mu_{\max } \rho C}{Y_{\mathrm{xs}}\left(K_{\mathrm{M}}+C\right)}
$$

where Monod growth kinetics have been assumed. Note that the parameters $D_{\mathrm{e}}$ and $\rho$ are allowed to vary with $x$ and $y .{ }^{44,45} \rho=\rho(x, y)$ is the local cell density, which is obtained based on the experimental greyscale image as follows. At each pixel, we assign a cell density proportional to the light intensity of the image such that the average cell density is $\rho$. If the total number of pixels is $N$, and the ith pixel intensity is $l_{i}$, then the cell density at the ith pixel is given by

$$
\rho_{i}=\rho\left(x_{i}, y_{\mathrm{i}}\right)=\frac{l_{\mathrm{i}} N}{\sum_{i=1}^{N} l_{\mathrm{i}}} \rho .
$$

We note that $\rho$ depends on space only and in the bulk fluid $\rho_{\mathrm{i}}=0$ since $l_{\mathrm{i}}=0$. 
The diffusion coefficient is given by

$$
D=\left\{\begin{array}{c}
D_{\mathrm{e}}, \text { in biofilm } \\
D_{\mathrm{b}}, \text { in bulk fluid }
\end{array}\right.
$$

where $D_{\mathrm{b}}$ is an apparent diffusion coefficient that has been artificially elevated so as to account for convective mixing that occurs in the moving fluid adjacent to the biofilm. $D_{\mathrm{e}}$ is the effective diffusive permeability of glucose inside the biofilms which depends on space only. $D_{\mathrm{e}}=D_{\mathrm{e}}(x, y)$ is calculated based on the approach derived by Hinson and Kocher ${ }^{46}$ as outlined in (ref. 47), and expressed by the equations below. In this formulation, $D_{\mathrm{eo}}$ is the effective diffusive permeability of the extracellular matrix, $D_{\mathrm{c}}$ is the diffusive permeability of the cell phase and $D_{\mathrm{p}}$ is the diffusive permeability of the pure extracellular polymer. $\epsilon_{\mathrm{aq}}, \epsilon_{\mathrm{c}}$ and $\epsilon_{\mathrm{p}}$ denote the volume fractions of water, cells and extracellular polymers, respectively. Here at the ith pixel, the cell volume fraction is $\epsilon_{\mathrm{C}}=\rho / \rho_{\mathrm{in}}$ where $\rho_{\text {in }}$ is the cell intrinsic density.

$$
\begin{aligned}
& \frac{D_{\mathrm{e}}}{D_{\mathrm{aq}}}=\left(\frac{D_{\mathrm{e}}}{D_{\mathrm{eo}}}\right)\left(\frac{D_{\mathrm{eo}}}{D_{\mathrm{aq}}}\right) \\
& \frac{D_{\mathrm{e}}}{D_{\mathrm{eo}}}=\frac{2 \frac{D_{\mathrm{aq}}}{D_{\mathrm{c}}}+\frac{D_{\mathrm{aq}}}{D_{\mathrm{eo}}}-2 \epsilon_{\mathrm{c}}\left(\frac{D_{\mathrm{aq}}}{D_{\mathrm{c}}}-\frac{D_{\mathrm{aq}}}{D_{\mathrm{eo}}}\right)}{2 \frac{D_{\mathrm{aq}}}{D_{\mathrm{c}}}+\frac{D_{\mathrm{aq}}}{D_{\mathrm{eo}}}+\epsilon_{\mathrm{c}}\left(\frac{D_{\mathrm{aq}}}{D_{\mathrm{c}}}-\frac{D_{\mathrm{aq}}}{D_{\mathrm{eo}}}\right)} \\
& \frac{D_{\mathrm{eo}}}{D_{\mathrm{aq}}}=\epsilon_{\mathrm{aq}}\left(\epsilon_{\mathrm{p}} \frac{D_{\mathrm{aq}}}{D_{\mathrm{p}}}+\epsilon_{\mathrm{aq}}\right)^{-1} \\
& b=\frac{\epsilon_{\mathrm{p}}}{\epsilon_{\mathrm{c}}+\epsilon_{\mathrm{p}}} \\
& \epsilon_{\mathrm{aq}}+\epsilon_{\mathrm{c}}+\epsilon_{\mathrm{p}}=1
\end{aligned}
$$

The boundary conditions are

$$
\begin{aligned}
& \frac{\partial C}{\partial x}=0 \text { for } 0<y<Y \text { at } x=0 \text { and } x=X \\
& \frac{\partial C}{\partial y}=0 \text { for } 0<x<X \text { at } y=0 \\
& C=C_{0} \text { for } 0<X<X \text { at } y=Y
\end{aligned}
$$

which stipulate no flux conditions along the bottom and sides and a constant concentration along the top boundary.

The unsteady reaction-diffusion equation was solved on a rectangular spatial domain by the finite element method with discretisation in space and backward Euler difference in time. The computation was terminated when the relative change in $C$ between consecutive time steps was $<10^{-3}$, corresponding to a steady state. The microbial growth rate was calculated as

$$
\mu=\frac{\mu_{\max } C}{K_{\mathrm{M}}+C}
$$

where $C$ is the local steady state concentration of the growth-limiting substrate.

Oxygen concentration profiles in colony biofilms

These methods pertain to Case 1. The Case 1 assumption of zero-order reaction kinetics is an appropriate approximation when the Monod halfsaturation coefficient for the substrate is smaller than the concentration at the surface of the biofilm. This is the case for consumption of oxygen by Pseudomonas aeruginosa when the oxygen concentration at the biofilm interface is in equilibrium with air. ${ }^{48,49}$ Pseudomonas aeruginosa FRD1 colony biofilms were grown for $48 \mathrm{~h}$ and profiled with oxygen microelectrodes as previously detailed. ${ }^{50}$ To perform the experiment in an atmosphere enriched in oxygen, two holes were cut in the plastic lid of a Petri dish that was placed over the tryptic soy agar plate on which the colony biofilm rested. One hole was at the perimeter of the dish and allowed a silicone tube to be inserted, through which pure oxygen was delivered in a continuous stream. The second hole was centered in the lid and directly above the colony biofilm. Oxygen gas exited from this hole, which also enabled microelectrode access to the biofilm. A single-colony biofilm was first profiled in air, then flooded with oxygen gas as described above for $15 \mathrm{~min}$ and profiled again in this oxygen-enriched environment. The measurements were performed in the same spot in the biofilm (in duplicate in air and triplicate in oxygen). The oxygen concentration in equilibrium with air was measured to be $6.0 \mathrm{mg} \mathrm{I}^{-1}$ and with the oxygenenriched environment was $28.5 \mathrm{mg} \mathrm{I}^{-1}$.

The parabolic solution given by Equation (5) was fitted to experimentally measured concentration profiles by least squares regression to estimate $\phi_{\mathrm{o}}$ with the value of $C_{\mathrm{o}}$ fixed at the experimental value measured in the gas phase. A $t$-test of statistically significant difference between the values of $\phi_{\mathrm{o}}$ determined in air and oxygen was implemented for the non-linear least squares problem. ${ }^{51}$

Spatial pattern of GFP induction in flow-cell biofilms

These methods pertain to Case 3. Pseudomonas aeruginosa biofilms were grown in $1 \mathrm{~mm}$ square glass capillaries as described previously. ${ }^{52}$ Strain PAO1 containing plasmid PAB1 (ref. 53) was used. This strain carries a stable GFP under the control of the trc promoter, which is inducible with isopropylthio- $\beta$-D-galactoside. The minimal growth medium contained: $5 \mathrm{mmol} \mathrm{I}^{-1}$ glycerol, $0.9 \mathrm{mmol} \mathrm{I}^{-1}$ sodium glutamate, $145 \mathrm{mmol} \mathrm{I}^{-1} \mathrm{NaCl}$, $3.4 \mathrm{mmolI}^{-1} \mathrm{~K}_{2} \mathrm{HPO}_{4}, 1.5 \mathrm{mmol}^{-1} \mathrm{NaH}_{2} \mathrm{PO}_{4} \cdot 2 \mathrm{H}_{2} \mathrm{O}$ and $0.2 \mathrm{mmoll}^{-1}$ $\mathrm{MgSO}_{4} \cdot 7 \mathrm{H}_{2} \mathrm{O}$. Biofilms were grown under continuous flow at ambient temperature $\left(23^{\circ} \mathrm{C}\right)$ for 5 days prior to induction with $1 \mathrm{mmol}^{-1}$ isopropylthio- $\beta$-D-galactoside. Some biofilms were counterstained with $50 \mathrm{mg} \mathrm{l}^{-1}$ rhodamine $\mathrm{B}$ for $30 \mathrm{~min}$ after the induction step to reveal biomass that did not express GFP. Images were collected with a Leica TCS-NT confocal scanning laser microscope using a 488-nm laser for excitation of GFP and a 561-nm laser for excitation of rhodamine B. Quantitative image analysis was performed using the Linescan function of MetaMorph software (Molecular Devices, Sunnyvale, CA, USA).

The data analysis from these experiments began by extracting an estimate of the first-order Thiele modulus, $\phi_{1}$, by dividing the GFP intensity at the center of a cell cluster by the intensity at the cluster edge. This ratio corresponds to the value of Equation (18) in the limit as $r$ or $\xi$ goes to zero, which is $1 / \sinh \phi_{1}$. Thiele modulus values thus extracted were plotted versus cluster radius. From Equation (17), the slope of this line is $\left(k_{1} / D_{\mathrm{e}}\right)^{1 / 2}$. Then $k_{1, \mathrm{ex}}$ was found by multiplying the slope, determined by least squares regression, times the independently estimated effective diffusion coefficient.

\section{FISH of endocarditis specimens}

These methods pertain to Case 4B. Heart valve tissue was fixed immediately after excision in the operating room, embedded in methacrylate and sectioned as described before. ${ }^{54}$ The heart valve of the endocarditis patient was investigated by FISH and 16 S rRNA-gene-PCR and sequencing as part of the diagnostic routine workup of endocarditis. This use was exempt from human subjects approval and the related image included in the present study was fully anonymised. FISH was carried out using the Streptococcusspecific probe STREP1-Cy3 (false coloured red in Figure 5) ${ }^{55}$ combined with unspecific nucleic acid stain DAPI (4',6- diamidino-2-phenylindole dihydrochloride) to visualise all bacterial cells (false coloured blue in Figure 5). ${ }^{56}$ Microscopic examination of the tissue sections was performed using an epifluorescence microscope (AxioPlan II, Zeiss, Jena, Germany) equipped with a $100 \mathrm{~W}$ high-pressure mercury lamp and narrow band filtre sets. Image acquisition was performed with an AxioCam MRm black and white camera and the AxioVision 4.6 software. Consecutive sections were submitted to DNA extraction followed by broad-range $16 \mathrm{~S}$ rRNA-gene-PCR and sequencing as described. ${ }^{57}$

\section{ACKNOWLEDGEMENTS}

This work was supported by NIH/NIGMS award R01GM109452.

\section{CONTRIBUTIONS}

P.S.S. derived mathematical models, designed the research and wrote the manuscript. T.Z. performed numerical solution of 2D mathematical models. R.X., B.P., M.C.W. and F.R. performed in vitro experiments. A.M. and J.K. performed ex vivo FISH analysis.

\section{COMPETING INTERESTS}

The authors declare no conflict of interest. 


\section{REFERENCES}

1. LaMotta, E. J. Internal diffusion and reaction in biological films. Environ. Sci. Technol. 10, 765-769 (1976).

2. Williamson, K. \& McCarty, P. L. A model of substrate utilization by bacterial films. J. Water Pollut. Control Fed. 48, 9-24 (1976).

3. Baltzis, B. C. \& Fredrickson, A. G. Competition of two microbial populations for a single resource in a chemostat when one of them exhibits wall attachment. Biotechnol. Bioeng. 25, 2419-2439 (1983).

4. Kissel, J. C., McCarty, P. L. \& Street, R. L. Numerical simulation of mixed-culture biofilm. J. Environ. Eng. 110, 393-411 (1984).

5. Wanner, O. \& Gujer, W. A multispecies biofilm model. Biotechnol. Bioeng. 28, 314-328 (1986).

6. Rittmann, B. E. \& Manem, J. A. Development and experimental evaluation of a steady-state, multispecies biofilm model. Biotechnol. Bioeng. 39, 914-922 (1992).

7. Kreft, J.-U., Booth, G. \& Wimpenny, J. W. T. BacSim, a simulator for individualbased modelling of bacterial colony growth. Microbiology 144, 3275-3287 (1998).

8. Picioreanu, C., van Loosdrecht, M. C. M. \& Heijnen, J. J. Mathematical modeling of biofilm structure with a hybrid differential-discrete cellular automaton approach. Biotechnol. Bioeng. 58, 101-116 (1998).

9. Xavier, J. B., Picioreanu, C. \& van Loosdrecht, M. C. M. A framework for multidimensional modelling of activity and structure of multispecies biofilms. Environ. Microbiol. 7, 1085-1103 (2005).

10. Costerton, J. W., Stewart, P. S. \& Greenberg, E. P. Bacterial biofilms: a common cause of persistent infections. Science 284, 1318-1322 (1999).

11. Parsek, M. R. \& Singh, P. K. Bacterial biofilms: an emerging link to disease pathogenesis. Annu. Rev. Microbiol. 57, 677-701 (2003).

12. Hall-Stoodley, L. \& Stoodley, P. Evolving concepts in biofilm infections. Cell Microbiol. 11, 1034-1043 (2009).

13. Bjarnsholt, T. The role of bacterial biofilms in chronic infections. APMIS Suppl. 121, 1-51 (2013).

14. Nichols, W. W., Evans, M. J., Slack, M. P. E. \& Walmsley, H. L. The penetration of antibiotics into aggregates of mucoid and non-mucoid Pseudomonas aeruginosa. J. Gen. Microbiol. 135, 1291-1303 (1989).

15. Dibdin, G. H. Plaque fluid and diffusion: study of the cariogenic challenge by computer modeling. J. Dent. Res. 69, 1324-1331 (1990).

16. Stewart, P. S. Biofilm accumulation model that predicts antibiotic resistance of Pseudomonas aeruginosa biofilms. Antimicrob. Agents Chemother. 38, 1052-1058 (1994).

17. Stewart, P. S. Theoretical aspects of antibiotic diffusion into microbial biofilms. Antimicrob. Agents Chemother. 40, 2517-2522 (1996).

18. Roberts, M. E. \& Stewart, P. S. Modeling protection from antimicrobial agents in biofilms through the formation of persister cells. Microbiology 151, 75-80 (2005).

19. Anguige, K., King, J. R. \& Ward, J. P. A multi-phase mathematical model of quorum sensing in a maturing Pseudomonas aeruginosa biofilm. Math. Biosci. 203, 240-276 (2006).

20. Chambless, J. D., Hunt, S. M. \& Stewart, P. S. A three-dimensional computer model of four hypothetical mechanisms protecting biofilms from antimicrobials. Appl. Environ. Microbiol. 72, 2005-2013 (2006).

21. Cogan, N. G. Incorporating toxin hypothesis into a mathematical model of persister formation and dynamics. J. Theor. Biol. 248, 340-349 (2007).

22. Eberl, H. J., Khassehkhan, H. \& Demaret, L. A mixed-culture model of a probiotic biofilm control system. Comput. Math. Methods Med. 11, 99-118 (2010).

23. Ilie, O., van Loosdrecht, M. C. \& Picioreanu, C. Mathematical modelling of tooth demineralisation and $\mathrm{pH}$ profiles in dental plaque. J. Theor. Biol. 309, 159-175 (2012).

24. Imran, M. \& Smith, H. L. A model of optimal dosing of antibiotic treatment in biofilm. Math. Biosci. Eng. 11, 547-571 (2014)

25. Kragh, K. N. et al. Polymorphonuclear leukocytes restrict growth of Pseudomonas aeruginosa in the lungs of cystic fibrosis patients. Infect. Immun. 82, 4477-4486 (2014).

26. Bailey, J. E. \& Ollis, D. F. Biochemical Engineering Fundamentals. 2nd edn, (McGrawHill, 1986).

27. Chen, P.-J. \& Faust, S. D. The solubility product of ferrous phosphate. Environ. Lett. 6, 287-296 (1974)

28. Price, W. E. \& Woolf, L. A. Intradiffusion coefficients for iron and water and shear viscosities in aqueous iron(II) perchlorate solutions at $25^{\circ} \mathrm{C}$. J. Solution Chem. 21, 239-247 (1992).

29. Stewart, P. S. Diffusion in biofilms. J. Bacteriol. 185, 1485-1491 (2003).

30. Wentland, E. J., Stewart, P. S., Huang, C.-T. \& McFeters, G. A. Spatial variations in growth rate within Klebsiella pneumoniae colonies and biofilm. Biotechnol. Prog. 12, 316-321 (1996).

31. Lehninger, A. L. Biochemistry. 2nd edn (Worth, 1975).
32. Margolis, D. J. et al. Lack of effectiveness of hyperbaric oxygen therapy for the treatment of diabetic foot ulcer and the prevention of amputation: a cohort study. Diabetes Care 36, 1961-1966 (2013).

33. Harremoës, P. Half-order reactions in biofilm and filter kinetics. Vatten 33, 122-143 (1977).

34. Worlitzsch, D. et al. Effects of reduced mucus oxygen concentration in airway Pseudomonas infections of cystic fibrosis patients. J. Clin. Invest. 109, 317-325 (2002).

35. Lone, A. G. et al. Staphylococcus aureus induces hypoxia and cellular damage in porcine dermal explants. Infect. Immun. 83, 2531-2541 (2015).

36. Cowley, E. S., Kopf, S. H., LaRiviere, A., Ziebis, W. \& Newman, D. K. Pediatric cystic fibrosis sputum can be chemically dynamic, anoxic, and extremely reduced due to hydrogen sulfide formation. mBio 6, e00767 (2015).

37. Borriello, G. et al. Oxygen limitation contributes to antibiotic tolerance of Pseudomonas aeruginosa in biofilms. Antimicrob. Agents Chemother. 48, 2659-2664 (2004).

38. Meyer, J. M., Neely, A., Stintzi, A., Georges, C. \& Holder, I. A. Pyoverdin is essential for virulence of Pseudomonas aeruginosa. Infect. Immun. 64, 518-523 (1996).

39. Stewart, P. S. \& Costerton, J. W. Antibiotic resistance of bacteria in biofilms. Lancet 358, 135-138 (2001).

40. Crank, J. The Mathematics of Diffusion. 2nd edn (Clarendon Press, 1975).

41. Hill, C. G. Jr. An Introduction to Chemical Engineering Kinetics \& Reactor Design. (John Wiley \& Sons, 1977).

42. Picioreanu, C., Kreft, J. U. \& Van Loosdrecht, M. C. Particle-based multidimensional multispecies biofilm model. Appl. Environ. Microbiol. 70, 3024-3040 (2004).

43. Petroff, A. P. et al. Reaction-diffusion model of nutrient uptake in a biofilm: theory and experiment. J. Theor. Biol. 289, 90-95 (2011).

44. Beyenal, H. \& Lewandowski, Z. Internal and external mass transfer in biofilms grown at various flow velocities. Biotechnol. Prog. 18, 55-61 (2002).

45. Van Wey, A. et al. Anisotropic nutrient transport in three-dimensional single species bacterial biofilms. Biotechnol. Bioeng. 109, 1280-1292 (2012).

46. Hinson, R. K. \& Kocher, W. M. Model of effective diffusivities in aerobic biofilms. J. Environ. Eng. 122, 1023-1030 (1996).

47. Stewart, P. S. A review of experimental measurements of effective diffusive permeabilities and effective diffusion coefficients in biofilms. Biotechnol. Bioeng. 59, 261-272 (1998).

48. Beyenal, H., Chen, S. N. \& Lewandowski, Z. The double substrate growth kinetics of Pseudomonas aeruginosa. Enzyme Microb. Technol. 32, 92-98 (2003).

49. Tanase, C., Chirvase, A. A., Ungureanu, C., Caramihai, M. \& Muntean, O. Study of double-substrate limited growth of Pseudomonas aeruginosa in aerobic bioprocess. Revue Roumaine Chemie 56, 1147 (2011).

50. Walters, M. C., Roe, F., Bugnicourt, A., Franklin, M. J. \& Stewart, P. S. Contributions of antibiotic penetration, oxygen limitation, and low metabolic activity to tolerance of Pseudomonas aeruginosa biofilms to ciprofloxacin and tobramycin. Antimicrob. Agents Chemother. 47, 317-323 (2003).

51. Kutner, M. H., Nachtsheim, C., Neter, J. \& Li, W. Applied Linear Statistical Models. (McGraw-Hill Irwin, 2005).

52. Klayman, B. J., Klapper, I., Stewart, P. S. \& Camper, A. K. Measurements of accumulation and displacement at the single cell cluster level in Pseudomonas aeruginosa biofilms. Environ. Microbiol. 10, 2344-2354 (2008).

53. Werner, E. et al. Stratified growth in Pseudomonas aeruginosa biofilms. Appl. Environ. Microbiol. 70, 6188-6196 (2004).

54. Mallmann, C. et al. Fluorescence in situ hybridization to improve the diagnosis of endocarditis: a pilot study. Clin. Microbiol. Infect. 16, 767-773 (2010).

55. Gescher, D. M. et al. Fluorescence in situ hybridisation (FISH) accelerates identification of Gram-positive cocci in positive blood cultures. Intl. J. Antimicrob. Agents 32, S51-S59 (2008).

56. Schmiedel, D. et al. Fluorescence in situ hybridization for identification of microorganisms in acute chorioamnionitis. Clin. Microbiol. Infect. 20, 0538-0541 (2014).

57. Gescher, D. et al. A view on Bartonella quintana endocarditis. Diagn. Microbiol. Infect. Dis. 60, 99-103 (2008).

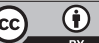

This work is licensed under a Creative Commons Attribution 4.0 International License. The images or other third party material in this article are included in the article's Creative Commons license, unless indicated otherwise in the credit line; if the material is not included under the Creative Commons license, users will need to obtain permission from the license holder to reproduce the material. To view a copy of this license, visit http://creativecommons.org/licenses/ by/4.0/

(c) The Author(s) 2016

Supplementary Information accompanies the paper on the npj Biofilms and Microbiomes website (http://www.nature.com/npjbiofilms) 